\title{
From Ontic Structural Realism
}

\author{
to \\ Metaphysical Coherentism
}

\author{
Matteo Morganti \\ University of Rome TRE
}

Forthcoming in European Journal for Philosophy of Science

Please only quote from the published version

\begin{abstract}
The present paper argues that the typical structuralist claims according to which invariances, symmetries and the like are fundamental - especially in quantum physics - are best understood not in terms of physical relations being fundamental, but rather in terms of 'metaphysical coherentism' - the view according to which object-like parts of reality exhibit symmetric relations of ontological dependence. The coherentist perspective is developed in some detail and contrasted with extant varieties of structuralism and holism.
\end{abstract}

\section{Introduction}

Structural realism (SR) attempts to re-establish the connection between the success of scientific theories and their probable truth, threatened by considerations having to do with the patent discontinuities that characterise the history of science. It does so by pointing at the structural continuity that exists between (some parts of some) subsequent theories across theory-change. SRists propose that we take such preserved structure as the (approximately) true part of the relevant theories. Epistemic structural realists conceive this as an epistemological position, to the effect that we can be realist about the relations that are described by the (preserved) formal structure of our theories. Ladyman [1998] introduced instead the 'ontic' variant of SR (OSR), according to which not only is structure all we can be realist about, but also all there is at the fundamental level. OSRists contend that this move allows one to fill an existing gap between epistemology and metaphysics, and to put indications coming from physical theory and from the history of science together in a virtuous consilience. 
This view has become increasingly popular in recent times, no doubt because it resonates with the way in which physics, in particular, has developed. However, OSR raises a number of worries, regarding both a) the justification for the move from epistemology to metaphysics and b) the workability of the underlying thesis about the nature of reality. In the present paper, I will take for granted that there are indeed good reasons for endorsing OSR, and will consequently focus on the second family of concerns. In what sense does contemporary science, especially physics, invite us to think about reality in structural terms? What is the resulting metaphysics, exactly?

Based on the plausible idea that, independently of issues of reduction, quantum theory is the part of science one should look at if one aims for a scientifically grounded metaphysical account of material reality, the literature has prevalently discussed the prospects for structuralism in the quantum domain. I will follow this trend. However, I will end up arguing that, given the present state of the debate and the limits that OSR turns out to have, the seemingly correct structuralist insights concerning quantum physics are best preserved by turning (back) to an object-oriented ontology. At the same time, I will acknowledge that at least some essential features of objects are, or may be, irreducibly extrinsic. While this may look like a more or less familiar sort of metaphysical holism, I will argue that, once it is aptly articulated, it is not. I will suggest that what structuralists correctly noticed about contemporary physics, and in particular quantum theory, is to be cashed out in terms of the relevant objects entering relations of mutual ontological dependence; and that the resulting view - which I will call 'metaphysical coherentism' - is arguably new, at least in its detailed formulation.

In Section 2, the typical structuralist contentions concerning quantum physics are briefly rehearsed, with the aim of identifying the sort of facts that any metaphysics presented as alternative to extant formulations of OSR must take into account and explain. ${ }^{1}$ Section 3 moves to the key problem, that of formulating a truly structuralist ontology of the quantum domain, and illustrates the limits of the proposals that have been considered in the literature so far. In Section 4, metaphysical coherentism is presented in detail. It is argued that not only is it a respectable metaphysical option; independently of the different ways in which it could be further developed, it in fact represents the best framework for flashing out the ideas that led to the formulation of OSR.

\footnotetext{
${ }^{1}$ Importantly, I will only consider structuralist arguments to the effect that physics positively urges us to attribute fundamental status to relational structure rather than objects. I will ignore a different strategy - employed by Ladyman in his earlier work and currently pursued mainly, if not uniquely, by Steven French: that of arguing in favour of structures on the basis of an under-determination of metaphysics by physics, in particular with respect to whether physical objects are individuals or non-individuals. The reason for this is that there is general agreement on the fact that a) based (mostly, but not exclusively) on reflections concerning the 'weak discernibility' of quantum particles, the recent literature has provided grounds for breaking the under-determination one way or another; yet, b) these developments lend support to the idea that relations in some sense 'come first' - in the case at hand, because quantum particles are at most differentiated one from the other by irreflexive symmetric relations that are not reducible to putative intrinsic features of the particles themselves.
} 
The coherentist view is also compared with more or less recent 'holistic' interpretations of quantum entangled systems. A short concluding section follows.

\section{Structuralism and Quantum Mechanics}

A key fact highlighted by OSRists is that in fundamental physics symmetry considerations play a key role, and one should pay particular attention to mathematical entities, known as 'groups', that capture the relevant symmetries. ${ }^{2}$ Indeed, according to structuralists, objects are to be reconceptualised "as representations of symmetry groups, where the symmetries reflect spatiotemporal, i.e. external, and internal degrees of freedom as well as permutation invariance" (Lyre [2004]; 662). Take for instance the Galilei group, the series of transformations that relate classical reference frames through translations, rotations and transitions from a frame to another which is in uniform motion with respect to the first. OSRists note that classical particles may be reconstructed in terms of the invariant representations of this group whose states are not decomposable into sub-states which are equally invariant under the relevant transformations - so called irreducible representations. Associated to these, there is an algebra of a specific type (a Lie algebra $^{3}$ ) which has certain invariants (the 'Casimir invariants') that correspond to allegedly intrinsic, essential properties of particles, such as mass, energy and spin magnitude. Focusing on what could be regarded as the relativistic analogue of this, based on the Poincaré group, McKenzie similarly claims that "it thus makes sense in this context to regard the states from different [...irreducible representations] of this group to be states of different physical systems. As such, it was proposed that different irreducible representations of the Poincaré group correspond to different species of relativistic particle.” (2014; sec. 6).

Thus, once one acknowledges the group-theoretic nature of physical theor ${ }^{4}$, and especially quantum theory, it becomes plausible to think that what we take to be fundamental 'bits of matter' in the intuitive, classical sense really are epiphenomenal manifestations of something rather different, such that relations between things, symmetries and invariances are more fundamental than the things (putatively) giving rise to those relations and exhibiting those symmetries and

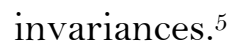

\footnotetext{
${ }^{2}$ More generally, physical theories exhibit 'external' space-time symmetries, and 'internal' gauge symmetries. The latter, in particular, entail a mismatch between the number of degrees of freedom the theory uses to describe a physical system at an instant in time, and the number of degrees of freedom required to derive predictions about the state of the physical system at other times. The physically meaningful degrees of freedom are then 'selected' as invariants under certain group transformations.

${ }^{3}$ The Galilei group is a Lie group with 10 parameters.

${ }^{4}$ Roberts [2011], for instance, explicitly speaks of 'group structural realism'.

${ }^{5}$ As an anonymous reviewer correctly notices, physical systems can also be represented in other ways, hence it may be argued that considerations concerning symmetries and groups do not lend particular support to OSR. Here, however, if only for the sake of argument, I will grant that one possible way of arguing in favour of OSR is based (also) on the sort of facts just illustrated.
} 
To this, one may object that irreducible representations correspond to types of particles considered at the abstract, formal level rather than to concrete particle-tokens. In response, structuralists can add (following, e.g., Varadarayan [1985], Mittelstaedt [1995] and Castellani [1998], the key idea being due to Murray, Von Neumann, Wigner and Mackey) that individual particles are picked out by identifying among the possible ones particular observable quantities, such as specific position or momentum values, via so-called systems of imprimitivity. Systems of imprimitivity are essentially collections of subsets determined by groups of permutations acting on a given set of elements under certain conditions. Here, they are to be conceived of as the tool that allows for the representation that has been picked out via irreducibility to be 'individuated' on the basis of (some of) the relevant physical properties. ${ }^{6}$

Against this background, the group $\mathrm{SU}(2) \times \mathrm{U}(1) \times \mathrm{SU}(3)$ can be regarded as a fundamental ontological basis, since it underpins the Standard Model of elementary particles, based on the Poincaré group and the electroweak and chromodynamic gauge groups. In particular, the $\mathrm{U}(1)$ group governs quantum electrodynamical entities and processes, the $\mathrm{SU}(2) \times \mathrm{U}(1)$ group 'gives rise' to those bosons that mediate electromagnetic and weak interactions (photons and $\mathrm{W}$ and $\mathrm{Z}$ bosons), and the $\mathrm{SU}(3)$ group determines the bosons that transmit the strong interactions described by quantum chromodynamics (i.e., the eight kinds of gluons). From this, a structuralist view of the ontology of fundamental physics follows.

Further support to OSR is supposed to come from another peculiar fact concerning the quantum domain: namely, entanglement. As is well-known, at least some quantum composite systems are entangled, which means, roughly, that their components are correlated in rather peculiar ways. For instance, the singlet state $\left.\left.\left.\left.\varphi=1 / \sqrt{2}(\uparrow\rangle_{1} \downarrow\right\rangle_{2}-\downarrow\right\rangle_{1} \uparrow\right\rangle_{2}\right)$ tells us that the system composed of particles 1 and 2 is such that its components will necessarily turn out to have opposite spin values in the given spatial direction upon measurement. Yet, taken separately, 1 and 2 both have the same probability of having spin up and spin down, which would entail that samespin outcomes for joint measurements should instead be possible. ${ }^{7}$ It is because of this sort of correlation that certain quantum systems violate the well-known Bell inequalities, and a conflict between quantum mechanics and relativity consequently arises. The peculiar nature of entanglement is explained by OSRists by arguing that the anti-correlations ('...has opposite spin to...') exhibited by entangled systems correspond to 'ontologically thick' relations, which are

\footnotetext{
${ }^{6}$ But, do we really obtain concrete particulars from imprimitivity systems, and more generally from local algebras, local applications of group operations etc.? Or just particular representations that remain at the abstract level? Also, how do we distinguish between different particle kinds 'governed' by the same groups? These are relevant questions we will get back to later.

${ }^{7}$ This description is sufficient for present purposes. For a much more informative treatment of entanglement, the reader is invited to look at Earman [2015]. As far as I can tell, the first explicit move from quantum entanglement to a structuralist ontology is made in Esfeld [2004].
} 
simply not present in the non-entangled case. These relations literally constitute the relevant physical systems, and are as ontologically basic as particles, if not more. Entanglement appears to be even more pervasive when one moves from non-relativistic quantum mechanics to quantum field theory and then to relativistic quantum field theory. This is particularly evident in so-called 'algebraic quantum field theory' (AQFT), a version of the theory that focuses on local algebras of operators rather than 'traditional' physical systems and observables. Lam [2013], for instance, claims that the sort of algebras employed in AQFT (technically, type-III algebras) are such that entanglement is intrinsically global and irreducible. ${ }^{8}$ This is further grist to the structuralist mill, of course, as putatively irreducible physical relations appear all the more essential as we move towards supposedly more fundamental descriptions of reality.

To complete the picture, a structuralist reading of space-time can be endorsed, according to which neither substantivalism (space-time points are fundamental substances) nor relationism (space-time relations are derivative on material objects) is correct, and it is instead the case that spatio-temporal relations are primitive and ontologically basic. ${ }^{9},{ }^{10}$

Having said this, let us now look at the attempts that have been made to turn OSR into a precise metaphysics, that is, to substantiate the claim that relational structure (be it grouptheoretic, entanglement or spatio-temporal structure) is ontologically basic.

\section{The Metaphysics of OSR}

OSR is in fact a family of philosophical views, sharing the abovementioned assumption that relational structure is (epistemically and) metaphysically fundamental. One possible classification is as follows ${ }^{11}$ :

a) Strong OSR: there is only relational structure, and objects can be dispensed with, as they are merely emergent or epiphenomenal entities;

b) Moderate OSR: objects and relations are on a par, as all properties are, at root, relational in nature, but objects also exist as (nothing but) "placeholders" in the relevant structures;

\footnotetext{
${ }^{8}$ To what extent entanglement should be regarded as ubiquitous in AQFT is, however, open to discussion. While Clifton and Halvorson [2001], for instance, argue that local operations can never destroy entanglement in the fieldtheoretic context, and thus there is a sense in which there are no independent subsystems in that domain, Valente [2013] disagrees.

${ }^{9}$ For a defence of such a position, see Muller [2011] (a reply to some more sceptical remarks by Wüthrich [2008]), but also Dorato [2000] and Dorato and Pauri [2006].

${ }^{10}$ In connection to this, as well as quantum statistics, considerations concerning permutation invariance play an important role. We'll get back to this later.

${ }^{11}$ OSRists sometime suggest (see, e.g., French [2014]; 201) that their view meshes naturally with the bundle theory according to which objects are nothing but collections of compresent, co-located or in any case co-existent propertyinstances. I am not convinced by this, as it seems to me that the extension of traditional bundle theory to the structuralist scenario is not straightforward, and something more complicated is required (for instance, along the lines of Mertz [2001]). At any rate, this issue is orthogonal to the one we are interested in here, and I will consequently ignore this particular option in what follows.
} 
c) Contextualist OSR: some properties of objects might be monadic and non-structurallyreducible, but objects are dependent on structure nevertheless, because identity facts are always contextual, i.e. extrinsically determined.

Strong OS is an eliminative metaphysics, according to which all there is (fundamentally) is physical relations. Moderate and contextualist OS, instead, do not eliminate objects, and 'just' give fundamental status to both objects and relations. Let us evaluate each option in turn.

\subsection{Strong OSR}

The eliminativist stance has been defended and developed most forcefully by French (see, for instance, French [2010] and [2014]). French grounds the ontological reduction of objects to relational structure in essential ontological dependence: something $x$ depends for its existence upon something else $y$, i.e., it is part of the essence of $x$ that $x$ exists only if $y$ exists. According to French, physics licenses the conclusion that objects only exist if the relevant structures exist; and the dependence is such that there is nothing to objects - intrinsic properties, identity, constitution, whatever - that is not cashed out, metaphysically speaking, in terms of structure. Whence, according to French, the plausibility of a reductionist form of physical structuralism.

However, a few problems arise. First, ontological reduction does not require just an 'only if existential claim, but also a stronger claim to the effect that what is essentially dependent can be eliminated without loss. But, as shown for instance by McKenzie [2014], either structures are defined extensionally, and objects are not eliminated; or they are defined intensionally, and we seem to lack a well-defined way of determining their identity conditions and, consequently, their connection with objects as derivative entities. Moreover, ontological dependence is generally not a relation that warrants reduction: that $a$ depends on $b$, as a matter of fact, presupposes the existence of both $a$ and $b$ (see Wolff [2012]; 620-1). Perhaps OSRists need to turn to a metaphysical relation different from ontological dependence? McKenzie [2014] makes the sensible point that supervenience is not a good candidate either, as it expresses co-variance of properties, without any accompanying claim about what comes first and what can or cannot be reduced to what. Thus, she (McKenzie [forthcoming]) suggests that OSRists should employ the notion of ontological determination, or grounding, as, unlike ontological dependence and supervenience, it warrants both a one-way direction of priority and the possibility of reduction of the determined to the determining.

Even granting this, here the key difficulty emerges. As Wolff [2012; 616-617,622] puts it, eliminativist OSRists must show that there is nothing else in the essence of objects beyond what is reducible to structure. But this is not the case: while structuralists (e.g., Muller [2009], [2015]) 
claim that all putative intrinsic, state-independent properties are in fact amenable to a grouptheoretic analysis, as a matter of fact different particle kinds, i.e., particles with different values of mass and spin, correspond to different irreducible representations of the same groups. This is tantamount to saying that at least some of the essential intrinsic features of the specific particles that populate the actual world are not structurally reducible. Indeed, in order to establish which ones among the particle-types that turn out to be possible in terms of irreducible representations are actual, one cannot but turn to empirical data. ${ }^{12}$

\subsection{Moderate OSR}

The difficulties just mentioned may push towards an alternative along the lines of option b) above. Indeed, McKenzie [2014] concludes her critical analysis by suggesting that moderate OSR is more plausible than strong OSR, in that it makes physical objects depend on structure but, at the same time, has it that there are actual entities that occupy the places determined by the relevant structures, thus avoiding the problematic reduction of everything to relations. The moderate position was originally proposed (by Esfeld and Lam [2006]) with a view to accounting for the nature of space-time in structuralist-yet-non-eliminativist terms, so steering clear of the recurrent "no relations without relata" objection: space-time points exist, the claim was, but they are nothing but the "bare occupants" of the relevant places in the spatiotemporal structure. Later, the view was turned into the more general thesis according to which objects cannot be eliminated, yet they have no intrinsic features whatsoever.

Interesting as the view may be, difficulties emerge for moderate OSR too. First, one may question the postulation of objects as 'mere matter', even more deprived of characterisation than Lockean bare particulars due to their lack of identity (see, e.g., Esfeld, Deckert and Oldofredi [forthcoming]). Secondly, following Lowe [2003], one may claim that ontological dependence is intimately connected to explanation ${ }^{13}$, and consequently cannot be symmetric - we will get back to this later. Third, one may suggest that, at least in the present case, mutual ontic dependence is sufficient for elimination: if $x$, a token of type A, exists if and only if $y$, a token of type B, exists, why not think that, in actual fact, i) either only $x$ or only $y$, i.e., either only category A or only category B, exist; or that ii) $x$ and $y$ are just aspects of something more fundamental, i.e., there is only one ontological category with 'aspects' corresponding to A and B? i) has simply not been regarded as a serious option by moderate OSRists, as it appears to lead back to either traditional

\footnotetext{
${ }^{12}$ On this, see McKenzie [forthcoming]. Worries concerning the connection between objects and structures in an eliminativist framework are also expressed by O'Conaill [2014].

${ }^{13}$ The connection of explanation appears even tighter if one assimilates - at least for present purposes - the notion of ontological dependence to that of ground, the latter having been discussed rather intensely as of late exactly as the realworld counterpart of metaphysical explanation (or, slightly differently, as the tool required to 'regiment' the notion of metaphysical explanation at the linguistic/conceptual level).
} 
object-oriented ontology or eliminative structuralism. As for ii), Esfeld and Lam [2011] themselves have indeed suggested that the distinction between objects and structures is merely conceptual, and what one finds at the fundamental level is objects - albeit as entities whose essential "ways of being" [sic] are entirely analysable in relational terms. This proposal, though, hardly qualifies as OSR any longer and, if anything, is rather reminiscent of a somewhat oldfashioned substance ontology.

The foregoing may not qualify as knock-down considerations against moderate OSR. Even so, however, it is important to recall that, as mentioned in the discussion of strong OSR, at least some intrinsic properties characterising particle-types appear not to be reducible to relational structure. If this is the case, of course, moderate OSR is simply not an option given the status of contemporary physical theory either, since it posits relations plus utterly property-less objects. Clearly, one could suggest that in view of the foregoing we should, so to speak, put some flash back on the 'thin' bones constituted by objects as these are conceived by moderate OSRists. This suggestion will indeed be taken seriously in a moment.

Before that, let me just briefly draw attention to two things to be borne in mind for the discussion to follow: i) the moderate OSRists' emphasis on symmetric relations of ontological dependence, and potential worries about such relations; and ii) the abovementioned idea that the only one-category metaphysical frameworks that deserve to be taken seriously are either eliminative structuralism or a traditional object-oriented ontology.

\subsection{Contextualist OSR}

According to some (mainly Ladyman [2007], but see also Stachel [2002]), it is really just identity facts that require - or, perhaps, allow for - a structuralist treatment. That identity is contextual, i.e., entirely extrinsic, is argued for essentially on the basis of the following reasoning:

a) If identity were primitive and intrinsic the truth of haecceitism (the doctrine that there can be differences between what distinct possible worlds say about certain individuals inhabiting them that do not correspond to overall qualitative differences between those worlds) would follow; but

b) Haecceitism is contradicted by contemporary science: in particular, our best theories of both material particles and space-time are permutation-invariant, i.e. assign no physical significance to exchanges of exactly similar particles or points; therefore,

c) The identity of the basic physical constituents of reality is not primitive and intrinsic to them. 
This may appear compelling. However, as for premise a), it is in fact not the case that primitive intrinsic identity entails haecceitistic differences. For, what is true of distinct worlds is not univocally determined by the nature of the identity of the objects that inhabit them. A counterpart-theoretic treatment of possible worlds, for instance, may allow one to assume primitive intra-world identities together with anti-haecceitism; and the same would be the case for some sort of 'Leibnizian super-essentialism'. With respect to b), permutation-invariance does not in fact entail the falsity of haecceitism: for, even if haecceitistic permutations were genuinely possible, this need not have empirically relevant consequences. ${ }^{14}$ Given the foregoing, it follows that c) too is questionable. This is an important open issue at the boundary between physics and the metaphysics of modality, and we cannot enter the details of it here. Nor do we need to. The important point for present purposes is just that the proposed inference from the permutation invariance exhibited by physical theory to contextual identity can be questioned. And * failing the argument concerning permutation-invariance/anti-haecceitism*, the detractor of OSR can plausibly say that there remains no compelling reason for endorsing contextualist OSR.

This appears all the more plausible once one notices that contextualist OSR departs in a nonnegligible manner from the initial intuition underpinning physical structuralism - which, recall, was that contemporary physics strongly suggests that the nature of things, and not only their identity, is essentially relational. Even if full-blown ontological dependence of objects on relational structure cannot be obtained, surely structuralists would like to have more than 'simply' identity being essentially a relational matter (of course, modulo constraints determined by the properties that are not amenable to structuralist reduction discussed above).

This, together with what was said concerning strong and moderate OS, means that, overall, the problem of turning the structuralist insights suggested by the historical development of science and by contemporary physics into a consistent, precise metaphysics is indeed a serious one.

\section{OSR and Metaphysical Coherentism}

What next? Let us, first of all, summarise the discussion so far as follows:

1) The features of contemporary science, especially quantum physics, point to the fact that relational structure is ontologically, not only epistemologically, basic;

\footnotetext{
${ }^{14}$ See, for example, the account of quantum statistics suggested in Morganti [2009a], according to which all statedependent properties of quantum particles in many-particle systems are holistic, or 'collective', properties that can only be consistently attributed to the system as a whole. Clearly, an exchange of particles would not make a statistically relevant difference in such a scenario.
} 
2) The primacy of relational structure suggests that networks of physical relations are prior to objects, and the latter should either be eliminated (radical OSR) or made extremely 'thin' (moderate or contextual OSR);

3) Neither radical, nor moderate, nor contextual OSR are truly compelling ontological frameworks.

One possible reaction to 3 ) is to question 1), so endorsing either an epistemic form of SR or a different variety of scientific realism (capable of answering the pessimistic meta-induction in a non-structuralist way). ${ }^{15}$ Alternatively, one could try to resist the conclusion implicit in the above, and reject 3). This is indeed the dialectics in the literature, with authors sympathetic to OSR trying to develop the corresponding metaphysics further along the lines that were critically assessed in the previous section, and enemies urging - in various ways - an abandonment of the entire project.

In the rest of the paper, I will instead suggest a different strategy. In particular, I will argue that the above stalemate does not force the metaphysically-inclined scientific realist to either i) stick to a traditional object-oriented ontology - so ignoring the important intimations that seem to come from contemporary physics; or ii) insist on structuralism in the hope that all properties, as well as identities and even the 'thinnest' of objects will eventually be reduced to relational structure - so turning a naturalistic thesis into a controversial conjecture concerning the putative state of future physics. ${ }^{16}$ As a matter of fact, I will contend, there is a third option that allows to effectively sidestep the issue: the rejection of 2 ) above and an ensuing reinterpretation of 1). The key underlying idea is that - contrary to what has been assumed so far - the 'structural' intimations of contemporary science, and especially quantum physics, do not necessarily point to the ontological primacy of physical relations, and can in fact be interpreted differently.

An initial hint in this sense comes from Linnebo [2008; 67-8], who explicitly takes into account the following possibility as formulated in the context of mathematical structuralism:

(ODO) Each object in D [where D is the domain of some mathematical structure] depends on every other object in D.

ODO is a holistic claim that rejects the commonsensical view that objects are independent of each other, and postulates instead a sort of 'global interconnectedness'. ${ }^{17}$ So far, this kind of view

\footnotetext{
${ }^{15}$ Obviously enough, one could also abandon scientific realism altogether.

${ }^{16}$ On this, see the compelling considerations made in McKenzie [forthcoming].

${ }^{17}$ On a related note, Ainsworth [2011; 81-83], based on an interpretation of Shapiro's mathematical structuralism, points out that structures may be prior to objects without relations also being prior, as one can have structures with objects but without relations in them.
} 
has been left virtually unexplored in the case of concrete objects. Wolff [2014;210], for instance, explicitly considers it, but ends up being skeptical that something like ODO can profitably be applied in the case of physics. As far as I understand, Wolff seems to have in mind the following. In the case of mathematics, ODO at least 'looks like' structuralism, thanks the fact that the relevant domains are in any case fully describable in structural terms, independently of what specific objects populate them. For instance, the objects we call complex numbers may be said to mutually depend on each other, yet be exhaustively analysed in terms of the complex field. In the case of physics, however, this is not the case: unless one makes objects essentially dependent on structure, one is in fact abandoning structuralism altogether, as traditional objects and intrinsic properties are ipso facto re-introduced and given ontological priority.

Obviously enough, the implicit thought here is that a proper explanation of certain physical facts can only be provided in a genuinely structural context, where the latter is intended as a context in which physical relations are ontologically prior. Independently of whether or not ODO can be interpreted as, or turned into, a full-blown version of physical structuralism (an issue I will get back to in the concluding part of the paper), the main claim that I wish to make in what follows is that this is incorrect: to the contrary, ODO constitutes a very good basis for understanding the facts about quantum physics that have been discussed in the previous sections. Let us, then, see in more detail how this may work.

\subsection{Metaphysical Coherentism ${ }^{18}$}

By far the most widespread view of the metaphysical structure of reality is foundationalism: the view that the world has a 'vertical', hierarchical structure and a fundamental level. The latter could be identified with the entire cosmos as a Parmenidean One or be somehow situated at an intermediate level (an as yet virtually unexplored but seemingly admissible alternative). However, the most popular form of metaphysical foundationalism is no doubt pluralistic foundationalism. In analogy with foundationalism in epistemology - where a 'dogmatic' basis of non-further justified reasons is invoked to justify our beliefs - this view has it that there is a multitude of fundamental constituents of reality which ground - directly or indirectly - everything else and are not themselves grounded. A paradigmatic example is, of course, the atomistic picture we are all familiar with, according to which there are a few kinds of basic entities with no internal structure, and everything is composed of them. ${ }^{19}$

\footnotetext{
${ }_{19}^{18}$ For further discussion of the ideas in this section, see Calosi and Morganti [forthcoming].

${ }^{19}$ While it seems obvious that part-whole relations give rise to well-founded, strict partial orders, the existence of a fundamental level of entities that do not have proper parts is not an axiom of mereology. Also, that there is a fundamental, all-encompassing whole, as presupposed by monists, is only an axiom in classical mereology, where unrestricted composition holds.
} 
Foundationalism has however been challenged, on the basis that it is incompatible with the possibility of endless chains of part-whole (or, more generally, dependence) relations, and that it is merely endorsed on the basis of commonsense intuition. A serious alternative could be a form of 'metaphysical infinitism', whereby infinite chains of dependence and priority are postulated, or at least admitted. This view could be invoked (and has, at least in passing and implicitly, been invoked - see, e.g., Ladyman and Ross [2007; 178]) by structuralists in support of their claim that there is no problem of relations without relata: this is because, their argument could go, relata are always analysable structurally, and it is 'relations all the way down', ad infinitum. Indeed, this is an important option for all those structuralists who do not share the thought that there must be a fundamental level of reality and, consequently, either a plurality of fundamental structures at the micro-level or a fundamental, unitary Structure identical with the Universe (see French [2014]).

Be this as it may, another view is relevant here: what I will from now on call 'metaphysical coherentism. As with coherentist solutions to the problem of justification in epistemology, metaphysical coherentism abandons the idea of a pyramidal structure of directed, asymmetric relations with an ultimate foundation. As in Quinean webs of beliefs, the entities that make up reality are instead deemed to be mutually related in structures that are at least partly composed by 'cycles' or 'loops'. This sort of view of metaphysical structure has already emerged erratically in the literature, but certainly calls for more systematic development and evaluation.

But, can coherentism be taken seriously at all? An obvious worry, mentioned while discussing moderate OSR, is that explanatory relations such as ontological dependence cannot be symmetric, on pain of vicious circularity - symmetry and transitivity leading to a violation of irreflexivity, hence to things ontologically depending themselves, and consequently acting as explanantia of their own existence/identity/nature. ${ }^{20}$ Coherentism does indeed appear to violate irreflexivity: since cycles of priority and dependence relations ultimately lead back to the starting point, it looks as though they necessarily make things self-dependent and self-explaining. In view of this, metaphysical coherentism does in fact appear to be a non-starter exactly in the same way in which, according to some, moderate OSR is.

However, this is not the end of the story. First, the idea that genuine metaphysical explanations must be based on irreflexive relations can be questioned (see, e.g., Fine [1995 $]^{21}$, Jenkins [2011] and Correia [2014]). Secondly, it is in fact not true that cycles entail the reflexivity of the relevant relations. As a matter of fact, irreflexivity may hold in a cycle, provided that one has either

\footnotetext{
${ }^{20}$ While, of course, everything depends on itself in a trivial sense, irreflexivity seems problematic in this particular case. For, what is at stake here are non-trivial relations underpinning genuinely informative metaphysical explanations: but, can we say that we actually explain an otherwise mysterious fact $a$ based on a distinct fact $b$, if in turn we explain $b$ on the basis of $a$ ?

${ }^{21}$ Here's Fine: "Two objects may [...] be taken to be reciprocally dependent [...] just in case they stand in an irreducible essential relationship, either together or in partnership with other objects." [Fine, 1995; 282-284].
} 
antitransitivity (the relevant relation is never transitive) or intransitivity proper (the relevant relation is not always transitive) ${ }^{22}$. In connection to the latter, think about quasi-transitivity as understood in social choice theory and microeconomics. The notion was introduced by Sen [1969] in order to account for the consequences of Arrow's theorem - according to which, when subjects have three or more distinct alternatives to choose from, no system can convert the ranked preferences of individuals into a shared, generally valid, ranking while meeting certain sensible criteria. Roughly, quasi-transitivity allows one to consistently have that (for a given subject) $a$ is not better than $b$, and $b$ is not better than $c$, and yet $c$ is better than $a$. Or, applied for instance to the sorites paradox, it enables one to consistently claim that $x$ is not more a heap than $y, y$ is not more a heap than $z$, and yet $z$ is more a heap than $x$. In our case, mutatis mutandis, one may analogously have that $a$ is prior to $b, b$ is prior to $c$, but $c$ is not prior to $a$. In the case in which $a$ is identical to $c$, one obtains the desired result, i.e., a structure or sub-structure in which everything depends (or may depend) on everything else except itself.

Suppose, however, that the coherentist wishes to admit cycles. One option is to claim that a more general, possibly quasi-transitive, relation 'coexists' with another one which is transitive and reflexive, hence possibly cyclic. In particular, one may postulate that entities that are next to each other in the cycle fully depend one on the other, while entities that are further away from each other in the cycle only enter relations of partial dependence. ${ }^{23}$ It could be, that is, that $b$ depends entirely on $a, c$ depends entirely on $b$, yet $c$ only depends partially on $a$, and does so exactly in virtue of the fact that - to shift for a moment to the vocabulary of grounding - a fully grounds $c$ s full ground but does not ground $c$ directly. If $a$ is then fully dependent on $c$, one obtains that $a$ only partially depends on itself, which might be just fine. For example, one may have that $a$ is selfdependent only to the extent that it grounds some aspects $\mathrm{A}, \mathrm{B}, \ldots$ of the entities that in turn determine some of its own features $\mathrm{P}, \mathrm{Q} \ldots$ - where, crucially, $\mathrm{A}, \mathrm{B} \ldots$ and $\mathrm{P}, \mathrm{Q} \ldots$ are distinct and not related by relations of entailment. This enables one to safely rule out trivial explanations of the form ' $x$ is $\mathrm{P}$ because $x$ is $\mathrm{P}$ '.

Alternatively, and this is perhaps the most plausible option, one can maintain that only (symmetric) partial dependence is relevant. ${ }^{24}$ In the particular case at hand, one can interpret this

\footnotetext{
${ }^{22}$ Transitivity is the claim that $\left.\forall x y z((\operatorname{Rxy} \wedge \mathrm{Ryz}) \Rightarrow \mathrm{Rxz})\right)$, and one could either negate the universal quantifier or the conditional that is quantified over.

${ }^{23}$ Talk of partial and full ground/ontological dependence has an intuitive meaning: either something is entirely (or not at all) dependent on something else for its own existence and/or properties, or it depends on that other thing but also on others.

${ }^{24}$ Intuitively, this may look like a non-starter, as full grounds must be found somewhere. But intuition is not, at least not obviously, a particularly helpful guide in philosophy. In the present case, our prejudices might be at least partly rooted in the fact that we tend to identify grounding/dependence relations with mereological relations - which indeed seem to give rise to fully transitive chains ultimately leading to entities and/or levels of reality that fully grounds (all the) other levels. However, first of all, part-whole relations represent just one possible form of ontological dependence, and it is far from obvious that they represent the natural choice in the case at hand. At the very least, there are reasons for
} 
in the sense that any entity in a given collection cooperates with all the other entities in the collection in determining the more complex, higher-level whole which in turn determines (some of) the features of each one of those entities. Thus, each entity partially depends on every other, and on the whole. And the whole partially depends on each particular entity. Obviously enough, this strongly suggests that each entity partially depends on itself, as it is among the entities the whole depends on, and in turn depends on the whole. ${ }^{25}$ However, as desired, full self-dependence is again ruled out.

Notice that talk of 'wholes' has now emerged alongside talk of 'cycles'. This is not by accident. To the contrary, it hints at an important analogy with a key idea underlying the most respectable forms of epistemological coherentism. In the epistemological case, 'sophisticated' coherentists have indeed rejected the charge of circularity by explaining that their view is - at root - one according to which the individual beliefs lend support to each other by contributing to the constitution of the holistic web or network that justifies each one of them. Something similar may hold in the metaphysical case. ${ }^{26}$ Indeed, it is pretty uncontroversial that the key insight underpinning physical structuralism is that each object is the very object that it is thanks to the fact that it belongs to the structure it in fact belongs to. (As we have seen, it turns out to also be the case that the relevant structure would be different if it did not connect the very entities it in fact connects - including, in particular, certain seemingly irreducible intrinsic properties). Quite importantly, this is, again, arguably non-trivial from the point of view of explanation. For, what we have here is not something of the form ' $x$ is $\mathrm{P}$ because $x$ is $\mathrm{P}$ ', but rather something like ' $x$ is $\mathrm{P}$, therefore Str is $\mathrm{S}$, therefore $x$ is R' - with $x$ being a random physical object, $\mathrm{P}$ and $\mathrm{R}$ two different properties of $x$, Str the structure to which $x$ belongs and S a property of Str. For instance, it could be that, say, a boson $b$ is an essential ingredient of the 'bosonic structure' that determines (part of) the properties of $b$ itself, where it is the fact that $b$ has certain state-independent, intrinsic properties that grounds

claiming that composition/decomposition relations in contemporary physics, especially quantum mechanics, are not amenable to an interpretation in terms of classical mereology (see, e.g., Healey [2013] and Caulton [2015]). Secondly, it is of course only when implemented in a foundationalist context that mereological relations guarantee a full ground. Having said this, full grounds are, on the other hand, easily identified once the idea is rejected that they must be 'external', 'at a deeper level' etc. with respect to the grounded entity: in a cycle of partial dependence $a-b-c-d-a \ldots$, for instance, $a$ is fully grounded by $a, b, c, d$ together. Note, though, that this does not contradict the claim in the main text, which is the claim that, so to put it, all the real work is done by partial dependence only. See also next footnote.

${ }^{25}$ It also entails that the whole fully depends on the plurality of the particular entities. But this is not problematic, as i) full dependence is entirely reducible to partial dependence; ii) the fact remains that, whenever less than the whole is considered, only partial dependence is relevant; iii) the whole may be fully dependent on the plurality of the entities that constitute it for its existence, while failing to be so dependent with respect to its properties; iv) there may not even be a whole in the sense of an entity additional to the components - all the relevant facts may just be collective facts about particular pluralities of entities. If anything, this helps see that metaphysical coherentism is not a form of monism more on this in the next section.

${ }^{26}$ While the sophisticated, holistic form of coherentism is what - I claim - one should have in mind here, notice that the strictly circular model is also relevant. Consider, for instance, the mutual dependence that is established between substrata and property instances on at least some accounts of the ontology of material objects; or, more to the point, the mutual dependence holding triplets of quarks together inside hadrons. 
the existence of the bosonic structure, and the latter only determines the state-dependent properties of $b$. A sort of 'holistic coherentism' consequently emerges. The key point, however, is not the existence of a putative whole in addition to the parts (which may in fact be consistently denied, as mentioned in footnote 25), but rather the key role played by the entire network of mutual ontological dependence relations.

In addition to the above, the holistic coherentist may wish to resort to something like quasireflexivity: something is related to (in our case, is partially dependent on/grounded in) itself if and only if it is related to (partially dependent on/grounded in) something other than itself. This is not essential, but appears useful, as something like this seems to be clearly the case if one has holistic networks of dependence. Also, it is relevant in the case of one-place structures, which represent an interesting limiting scenario. There, dependence relations, if they hold at all, hold trivially exactly in the same sense that it is trivial that $a=a$ for any $a$. It seems to me that this 'collapse' onto identity suggests that no real explanation is forthcoming in these cases. If so, one could opt for quasi-reflexivity and claim that, exactly because the structure is a one-object structure, the object constituting it is not structurally determined, but instead possesses the relevant features primitively. ${ }^{27}$

Whatever one thinks of the various notions and options above, at any rate, what is important to stress for the time being is that, once it is developed in some detail, the idea of mutual ontological dependence is by no means a non-starter. To the contrary, a form of metaphysical coherentism can be formulated that is both internally consistent and explanatorily powerful with respect to the relevant facts - in particular, those of quantum physics.

\subsection{Holisms}

Before moving on, it will be helpful to briefly compare metaphysical coherentism - in its sophisticated, holistic variant just discussed - with other forms of holism that have been presented in the literature, lest the former be regarded as a mere repetition of the latter.

First of all, metaphysical coherentism is different from the sort of relational holism put forward by Teller [1986]. On the basis of well-known facts that we have already mentioned, Teller suggests that in quantum mechanics there are at least some relations - most notably, entanglement relations - which are strongly non-supervenient on, hence irreducible to, the monadic properties of their relata. This, says Teller, entails that quantum mechanics violates particularism - the Lewisian doctrine that everything about physical systems is ultimately

\footnotetext{
${ }^{27}$ Which is not to say that it depends on itself. Personally, I share the intuition that a lone object cannot be informatively said to possess the identity that it has on structural grounds; but also the intuition that its identity is ungrounded in general. This means that it is more appropriate to postulate a primitive intrinsic identity for it. Whatever one makes of such intuitions, notice that a consequence of disagreement on this point is that one should accept the reflexivity of full ground/dependence, which - as we have seen - is not a negligible choice.
} 
analysable in terms of individual objects and their intrinsic properties - and is instead to be interpreted in terms of holistic, irreducible relations. While metaphysical coherentism agrees on this, it differs from Teller's relational holism in that: i) it makes a more general claim, not limited to entanglement; ii) it more explicitly takes everything relational to be understood in terms of ways - certified by our best current theories - in which physical objects 'exist together', that is, in terms of ontological dependence, rather than in terms of relations that are as physical, i.e., concrete, as objects.

Metaphysical coherentism is also different from the form of holism proposed by Ismael and Schaffer [forthcoming]. Again focusing primarily on entanglement, Ismael and Schaffer establish an analogy between common cause explanations and what they call 'common ground' explanations. On the basis of it, they conclude that the typical correlations and non-separability exhibited by entangled systems are best explained in terms of the entangled particles having the entire composite system as their ontological ground, determining the properties they may be detected to have, and the way in which they are interrelated. From this, Ismael and Schaffer move on to suggesting that the whole cosmos is an entangled whole and, as such, turns out to act as a common ground. Now, while this latter point arguably makes the form of holism put forward by Ismael and Schaffer as general and encompassing as metaphysical coherentism, there are also important differences.

First of all, Ismael and Schaffer take holism to entail, or at least strongly suggest, the ontological priority of the whole on its parts (monism), so inverting the intuitive direction of ontological dependence. Metaphysical coherentism doesn't do this, and is therefore at an advantage in terms of amount of conceptual revision requested. Also, Ismael and Schaffer subscribe to a Grounding Principle whereby, whenever non-identical entities $a$ and $b$ are modally connected, then either $a$ grounds $b$, or $b$ grounds $a$, or $a$ and $b$ are the joint result of some common ground $c$. Now, metaphysical coherentism also says that there is an entity - composed, in some sense of the term, of all the mutually dependent entities together - which determines (some of) the features of the composing entities. However, crucially, as mentioned earlier, this does not entail an ontological addition to the plurality of the composing entities: $a$ and $b$ are modally connected because $a$ grounds $b$ and $b$ grounds $a$, and talk of 'the whole' constituted by $a$ and $b$ may just be a shortcut replacing expressions such as ' $a$ and $b$ considered together as exhibiting mutual ontological dependence'. ${ }^{28}$ While coherentism is 'empirically equivalent' to Ismael and Schaffer's holism, then, it effectively avoids the need to subscribe to monism, as well as the (necessary) postulation of a third entity.

\footnotetext{
${ }^{28}$ In other words, there may just be a plurality $a b$ which is convenient, and easier, to refer to as a full-blown composite object when it comes to certain facts about it - facts which, however, are ultimately collective facts about $a$ and $b$ together.
} 


\subsection{Comparative Evaluation and Application}

Summing up, metaphysical coherentism denies that physical relations are prior to physical objects - or, at least, as fundamental as the latter. As per ODO above, it affirms that objects can be regarded as fundamental, provided one also acknowledges that an essential part of their being what they are does not derive from 'lower' or 'upper' more fundamental levels, and instead stems, so to put it, from 'horizontal', i.e., same-level, structures of mutual dependence relations with other objects. On this construal, depending on how the 'essential part' above is understood, two options emerge. Either i) OSR is to be conceived as a form of metaphysical coherentism, whereby (some of) the essential intrinsic properties and/or the identity of objects are determined by 'holistic' structures of the coherentist type; or ii) OSR is to be replaced by metaphysical coherentism, understood as a more traditional ontology where (at least some) essential properties or identities are not relationally determined yet, at the same time, it is agreed that objects are not completely autonomous with respect to their existence, essence or identity.

Given the dim prospects for a structuralist analysis of all intrinsic properties characterising all particle-types, I think it is undeniable that the second option is much more convincing as things stand today. As a matter of fact, it looks as though one may insist on option i) only by explicitly abandoning a widespread understanding of structuralism in terms of the fundamentality of (in this case, physical) relations rather than objects. Whether such reformulation truly differs from the replacement recommended by ii) may boil down to an otiose, mostly terminological question, so I will not discuss the issue further. ${ }^{29}$ Independently of this, at any rate, what is crucial here is that, to repeat, metaphysical coherentism can be turned into a credible and genuinely explanatory view; and, when it comes to putting it into practice, it delivers the same goods as OSR, but arguably without the shortcomings exhibited by the extant formulations of the latter.

Let us see this in more detail. First, coherentism is superior to eliminative OSR, in that it does not get rid of objects in the problematic attempt to make physical relations self-standing and fundamental, while reducing all putatively intrinsic properties. It is preferable to moderate OSR, because it establishes a form of mutual dependence between objects, and not between physical relations and 'pseudo-objects' actually deprived of intrinsic characterisation, avoiding in the way the postulation of a two-category ontology. Lastly, it is better than contextualist OSR because it does not put forward a claim exclusively about identity, and instead allows for more nuanced assertions, in agreement with the indications coming from our best current physics (which even the majority of structuralists does not regard as concerning only/essentially identity facts).

\footnotetext{
${ }^{29}$ Recall, however, the above discussion of ODO, mathematical structuralism and 'object-based structures'. There may also be an interesting connection with the notion of structure employed in the context of hylomorphic accounts of composite entities, but a discussion of it will have to wait for a future occasion.
} 
As for the application of metaphysical coherentism to physics, first, the (alleged) pervasiveness and irreducibility of entanglement can be accounted for in terms of mutual dependence relations among physical systems rather than in terms of physical relations additional to (and more basic than) those systems. As argued in the previous section and shown in more detail in Calosi and Morganti [forthcoming], in particular, it can be argued that entangled systems are composed of individual objects that, upon interaction, come to be mutually ontologically related with respect to their properties (properties which, not surprisingly, are then to be treated as holistic features of the whole and behave as such - for example from the point of view of statistics). The crucial point here is, of course, that the peculiar correlations typical of quantum entangled systems may be regarded as a direct consequence of (interactions determining) symmetric ontological dependence.

It is worth stressing, in this connection, that 'object' is to be understood in the loosest possible sense here, and the metaphysical coherentist is not forced to postulate an ontology of 'traditional' objects, such as, say, classical particles. In the context of AQFT, for instance, the relevant symmetric dependence relations may be said to hold among local algebras of observables rather than more or less traditional 'concrete things'. ${ }^{30}$ A coherentist analysis goes through nonetheless.

As for particle-types and the idea that the properties of every particular particle of each type are determined by the characteristics of the relevant groups, this can perfectly be interpreted in terms of holistic structures of mutual dependence of the coherentist type, where such structures exclusively involve physical objects and symmetric relations of dependence among them. Of course, as we have seen, whether this understanding of particle kinds is viable or not is to say the least unclear and in need of further work both at the philosophical and physical level. However, to the specific extent that particle-types are structurally determined (whatever this extent exactly is), it doesn't make a difference whether one understands this in terms of physical structures being prior to objects or in terms of objects being mutually related.. ${ }^{31}$ Yet, as argued, the latter option is advantageous for independent reasons and should, therefore, be preferred.

Metaphysical coherentism, notice, also has the potential for offering a consistent overall view of the quantum domain. For instance, instead of offering separate coherentist/structuralist stories in the case of, say, entanglement and particle-kinds, one could regard facts concerning particle-types as prior to facts about particle-tokens. Consequently, one could claim that the 'modal constraints' that follow from the mutual ontological dependence among particle-types include those that give rise to actual quantum correlations. For example, the Pauli Exclusion Principle, dictating that two

\footnotetext{
${ }^{30}$ Especially so if, as some have claimed, shifting the ontological focus to algebras is necessary in order to avoid the problem of inequivalent representations. For discussion of this point (and two rather different takes on it), see French [2013] ad Glick [2016].

${ }^{31}$ Importantly, the fact that concrete individuals are not eliminated removes the problem of 'extracting' particular, concrete objects out of general, abstract structures. Hence, there is no need in a coherentist context to have recourse to tools such as imprimitivity systems and the like, nor to ask whether these tools truly give us concrete particulars, or just particular, yet abstract, representations.
} 
'identical' fermions can only be in the same state if anti-correlated, might be said to follow from the fundamental features of those networks of mutual ontological dependence that characterise fermions in general, and that are expressed by the relevant groups. Actual cases of anti-correlation (i.e., entanglement) would then be regarded as particular instantiations of these modal facts holding generally at the level of particle-kinds. From this, the potential emerges for a unitary account of quantum ontology including the Standard Model, entanglement, (non-)separability, the seeming failure of Humean Supervenience - recall the discussion of Teller's relational holism above - and more. (This, it goes without saying, is just a suggestion which calls for further work in the future).

Moving briefly to a different context, metaphysical coherentism appears to be applicable also in the case of the ontology of space(-time). While moderate OSR about space-time claims that spacetime points and spatio-temporal relations are equally fundamental, and radical OSR regards spatio(-temporal) relations as ontologically prior, the coherentist states that only space-time points exist, and they are mutually dependent on each other for their existence and metric features. Spatio-temporal relations are instead dependent entities. To what extent and in what sense this qualifies as structuralism about space-time is, again, open to discussion. (For sure, at a first glance the suggested coherentist understanding appears closer to space-time substantivalism than to relationism). The key point is, however, once again, that whatever intimations the structuralist claims we should be ready to receive from our best physics, it looks like they can be readily translated into an object-based language and ontology, provided that these possess the holistic features based on mutual ontological dependence typical of coherentism. ${ }^{32}$

\section{Conclusions}

Let us wrap up. Many claims to the contrary notwithstanding, science does not urge us to turn to an ontology of irreducible physical relations. In fact, it could be contended, all structuralist talk (whatever structuralist claims one exactly takes to be plausible and supported by current science) can be translated in terms of specific sets of objects being interrelated and mutually ontologically dependent in such a way that:

\footnotetext{
${ }^{32}$ One may object that in the case of space-time there are no intrinsic features of points that are not analysable structurally, hence either there is no reason for abandoning moderate OSR, or coherentism is at any rate equivalent to moderate OSR. While it is true that in this particular case the distance between (moderate) OSR and coherentism is smaller than in the case of material objects, however, the differences remain that the latter postulates one ontological category only, while the former postulates two; that the latter, unlike the former, takes spatio-temporal relations to be fundamental; and that the latter, but not the former, might attribute primitive identities to space-time points.
} 
i) Their joint existence determines the existence of specific structures (of mutual dependence, not of physical relations! $\left.{ }^{33}\right)$;

ii) The resulting structures constrain at least some of the features of each particular element of the set.

Looking in particular at the quantum domain, I argued that endorsing metaphysical coherentism is the right move to make for the defender of OSR, or at any rate for those who wish to make sense of certain 'structuralist' insights suggested by contemporary physics. Indeed, the coherentist interpretation of OSR, or, perhaps more plausibly, the endorsement of metaphysical coherentism as a (more) scientifically respectable alternative to OSR, makes sense of the typical claims that science urges us to drop (at least partially) the hierarchy metaphor in favour of some form of interconnectedness, and that the role played by symmetries, mathematical groups, permutation invariance and the like at the theoretical level truly has ontological import. At the same time, the proposed view does not require one to substantiate the more contentious claims that all properties are structurally reducible, or that physical relations, somehow directly corresponding to group transformations, invariances etc., are ontologically fundamental in the sense of literally constituting physical reality. Further study of the consequences, assumptions and ramifications of metaphysical coherentism is certainly in order - for instance, with respect to the recent debates on Humean Supervenience, the nature of composition and non-locality, or in connection to quantum gravity, or the relationships between fundamental physics and other domains of scientific inquiry. For the time being, however, I hope that sufficient reasons have been provided here at least for regarding metaphysical coherentism as a serious option that naturalistic philosophers should explore further in the future. ${ }^{34}$

\section{References}

Ainsworth, P. [2011]: Ontic Structural Realism and the Principle of the Identity of Indiscernibles, Erkenntnis, 75, 67-84.

Calosi, C. [2013]: Quantum Mechanics and Priority Monism, Synthese, 191, 1-14.

Castellani, E. [1998]: Galilean Particles: An Example of Constitution of Objects, in Castellani, E. (ed.): Interpreting Bodies, Princeton University Press, 181-194.

\footnotetext{
${ }^{33}$ I stress again this point because it is crucial: were the coherentist to postulate objects and physical relations, his/her position would be indistinguishable, say, from the moderate OSRists'. The emphasis on ontological dependence entails instead that the basic ontological inventory may well include only objects, even though the whole story concerning their properties will not be told unless it will include some sort of mutual interrelations. The relevant relations will of course be physical in the sense that they are captured and described by physics in some way and at some level, but not in the sense of being as concrete as objects.

${ }^{34} \mathrm{I}$ am grateful for their valuable feedback to a few anonymous reviewers as well as audiences in Geneva, Milan, Paris and Rome. The paper has also benefited from discussions and exchanges I had with Ricki Bliss, Claudio Calosi and Emanuele Rossanese.
} 
Caulton, A. [2015]: Is Mereology Empirical? Composition for Fermions, in Wüthrich, C. and Bigaj, T. (eds.): Metaphysics in Contemporary Physics, Poznan Studies in the Philosophy of the Sciences and the Humanities, Rodopi, 293-321.

Clifton, R. and Halvorson, H. [2001]: Entanglement and Open Systems in Algebraic Quantum Field Theory, Studies in History and Philosophy of Modern Physics, 32, 1-31.

Correia, F. [2014]: Logical Grounds, The Review of Symbolic Logic, 7, 31-59.

Dorato, M. [2000]: Substantivalism, Relationism, and Structural Spacetime Realism, Foundations of Physics, 30, 1605-1628.

Dorato. M. and Pauri, M. [2006]: Holism and Structuralism in Classical and Quantum GR, in Rickles, D., French S. and Saatsi, J. (eds.): The Structural Foundations of Quantum Gravity. Clarendon Press, 121-151.

Earman, J. [2015]: Some Puzzles and Unresolved Issues About Quantum Entanglement, Erkenntnis, 80, 303-337.

Esfeld, M. [2004]: Quantum Entanglement and a Metaphysics of Relations, Studies in History and Philosophy of Modern Physics, 35, 601-617.

Esfeld, M. and Lam, V. [2006]: Moderate Structural Realism About Space-Time, Synthese, 160, 2746.

Esfeld, M. and Lam, V. [2011]: Ontic Structural Realism as a Metaphysics of Objects, in Bokulich, A. and Bokulich, P. (eds.): Scientific Structuralism, Springer Science+Business Media, 143-159.

Esfeld, M., Deckert, D.-A. and Oldofredi, A. [forthcoming]: What is Matter? The Fundamental Ontology of Atomism and Structural Realism, in Ijjas A. and Loewer, B. (eds.): A Guide to the Philosophy of Cosmology, Oxford University Press.

Fine, K. [1994]: Essence and Modality, in Tomberlin, J. (ed.): Philosophical Perspectives, Ridgeview Publishing, 1-16.

Fine, K. [1995]: Ontological Dependence, Proceedings of the Aristotelian Society, 95, 269-290.

French, S. [2003]: Scribbling on the Blank Sheet: Eddington's Structuralist Conception of Objects, Studies in History and Philosophy of Modern Physics, 34, 227-259.

French, S. [2010]: The Interdependence of Structure, Objects and Dependence, Synthese, 175, 89-109.

French, S. [2013]: Unitary Inequivalence as a Problem for Structural Realism, Studies in History and Philosophy of Modern Physics, 43, 121-136.

French, S. [2014]: The Structure of the World: Metaphysics and Representation, Oxford University Press.

Glick, D. [2016]: The Ontology of Quantum Field Theory: Structural Realism Vindicated?, Studies in the History and Philosophy of Modern Physics, 59, 78-86.

Healey, R. [2013]: Physical Composition, Studies in History and Philosophy of Modern Physics, 44, 48-62.

Ismael, J. and Schaffer, J. [forthcoming]: Quantum Holism: Nonseparability as Common Ground, Synthese.

Jenkins, C.S. [2011]: Is Metaphysical Dependence Irreflexive?, The Monist, 94, 267-276.

Ladyman, J. [1998]: What is Structural Realism?, Studies in History and Philosophy of Science, 29, 409-424.

Ladyman, J. [2007]: On the Identity and Diversity of Objects in a Structure, Proceedings of the Aristotelian Society, 81, 23-43.

Ladyman, J. and Ross, D. (with Spurrett, D. and Collier, J.) [2007]: Every Thing Must Go. Metaphysics Naturalised, Oxford University Press.

Lam, V. [2013]: The Entanglement Structure of Quantum Field Systems, International Studies in the Philosophy of Science, 27, 59-72.

Lowe, E. J. [2003]: Individuation, in Loux, M.J. and Zimmerman, D.W. (eds.): The Oxford Handbook of Metaphysics, Oxford University Press, 75-95.

Lyre, H. [2004]: Holism and Structuralism in U(1) Gauge Theory, Studies in History and Philosophy of Modern Physics, 35, 643-670. 
McKenzie, K. [forthcoming]: Structuralism in the Idiom of Determination, British Journal for the Philosophy of Science.

McKenzie, K. [2014]: Priority and Particle Physics: Ontic Structural Realism as a Fundamentality Thesis, British Journal for the Philosophy of Science, 65, 353-380.

Mertz, D.W. [2001]: Individuation and Instance Ontology, Australasian Journal of Philosophy, 79, $45-61$.

Mittelstaedt, P. [1995]: The Constitution of Objects in Classical Mechanics and in Quantum Mechanics, International Journal of Theoretical Physics, 34, 1615-1626.

Morganti, M. [2009]: Ontological Priority, Fundamentality and Monism, Dialectica, 63, 271-288.

Morganti, M. [2009a]: Inherent Properties and Statistics with Individual Particles in Quantum

Mechanics, Studies in History and Philosophy of Modern Physics, 40, 223-231.

Morganti, M. [2015]: Dependence, Justification and Explanation: Must Reality Be Well-Founded?, Erkenntnis, 80, 555-572.

Muller, F.A. [2009]: Withering Away, Weakly, Synthese, 180, 223-233.

Muller, F.A., [2011]: How to Defeat Wüthrich's Abysmal Embarrassment Argument Against SpaceTime Structuralism, Philosophy of Science, 78, 1046-1057.

Muller, F.A. [2015]: The Rise of Relationals, Mind 124, 201-237.

O'Conaill, D. [2014]: Ontic Structural Realism and Concrete Objects, The Philosophical Quarterly, 64, 284-300.

Roberts, B.W. [2011]: Group Structural Realism, British Journal for the Philosophy of Science, 62, 47-69.

Schaffer, J. [2010]: Monism: The Priority of the Whole, Philosophical Review, 119, 31-76.

Sen, A. [1969]: Quasi-Transitivity, Rational Choice and Collective Decisions, Review of Economic Studies, 36, 381-393.

Stachel, J. [2002]: The Relations Between Things' Versus 'the Things Between Relations': The Deeper Meaning of the Hole Argument, in Malament, D. (ed.): Reading Natural Philosophy: Essays in the History and Philosophy of Science and Mathematics, Open Court, 231-266.

Tahko, T.E. and O'Conaill, D. [2012]: On the Common Sense Argument for Monism, in Goff, P. (ed.): Spinoza On Monism. Palgrave Macmillan, 149-166.

Tegmark, M. [2014]: Our Mathematical Universe, Knopf.

Teller, P. 1986]: Relational Holism and Quantum Mechanics, British Journal for the Philosophy of Science, 37, 71-81.

Valente, G. [2013]: Local Disentanglement in Relativistic Quantum Field Theory, Studies in History and Philosophy of Modern Physics, 44, 424-432.

Varadarayan, V.S. [1985]: Geometry of Quantum Theory, Springer.

Wolff, J. [2012]: Do Objects Depend on Structures?, British Journal for the Philosophy of Science, 63, 607-625.

Wüthrich, C. [2009]: Challenging the Space-time Structuralist, Philosophy of Science, 76, 10391051. 